\title{
Case Report Form
}

National Cancer Institute

\section{Source}

National Cancer Institute. Case Report Form. NCI Thesaurus. Code C40988.

A printed, optical, or electronic document designed to record all of the clinical studyrequired information stipulated in the protocol to be reported to the sponsor on each clinical trial subject. 